Internat. J. Math. \& Math. Sci.

\title{
AN ELEMENTARY INEQUALITY
}

\author{
SEYMOUR HABER \\ United States Department of Commerce \\ National Bureau of Standards \\ Washington, D.C. 20234
}

(Received November 8, 1978)

ABSTRACT. An elementary inequality is proved in this note.

KEY WORDS AND PHRASES. Inequality, Compound interest.

AMS (MOS) SUBJECT CLASSIFICATION (1970) CODES. 26015.

1. INTRODUCTION AND RESULTS.

The following theorems present a series of closely related inequalities. The form that was found origina11y is given first.

THEOREM 1. If $\mathrm{a}, \mathrm{b} \geq 0$ and $\mathrm{n}$ is a positive integer, then

$$
\frac{a^{n}+a^{n-1} b+a^{n-2} b^{2}+\ldots+b^{n}}{n+1} \geq\left(\frac{a+b}{2}\right)^{n},
$$

PROOF. Let $[x]$ be, as usual, the integer part of $x$, and let the symbol $\sum_{0}^{[n / 2]} *$ be defined by : 


$$
\sum_{0}^{[n / 2] *} x_{i}=\left\{\begin{array}{l}
x_{0}+x_{1}+\ldots+x_{[n / 2]} \text { if } n \text { is odd } \\
x_{0}+x_{1}+\ldots+x_{[n / 2]-1}+\frac{1}{2} x_{[n / 2]} \text { if } n \text { is even. }
\end{array}\right.
$$

Assuming, without loss of generality, that $a \geq b$, we divide through by $a^{n}$ in $(1.1)$, and set $\mathbf{x}=b / a$, obtaining :

$$
\frac{1+x+x^{2}+\ldots+x^{n}}{n+1} \geq\left(\frac{1+x}{2}\right)^{n} ; 0 \leq x \leq 1
$$

Now

$$
(1+x)^{n}=\sum_{0}^{[n / 2]} *\left({ }_{i}^{n}\right)\left(x^{i}+x^{n-i}\right)
$$

and

$$
1+x+x^{2}+\ldots+x^{n}=\sum_{0}^{[n / 2]} *\left(x^{i}+x^{n-i}\right) ;
$$

so that we may rewrite $(1.2)$ as :

$$
\sum_{0}^{[n / 2]} *\left(\frac{1}{n+1}-\frac{1}{2^{n}}\left(\begin{array}{l}
n \\
i
\end{array}\right)\right)\left(x^{i}+x^{n-i}\right) \geq 0 ; 0 \leq x \leq 1,
$$

We now note that $1+x^{n} \geq x+x^{n-1} \geq x^{2}+x^{n-2} \geq \ldots \geq x^{[n / 2]}+x^{n-[n / 2]}$. This is an example of the rearrangements inequality $([1], \mathrm{p} .261)$, since we can write

$$
x^{k}+x^{n-k}=x^{k+1 / 2} x^{-1 / 2}+x^{n-k-1 / 2} x^{1 / 2}
$$

and

$$
x^{k+1}+x^{n-k-1}=x^{k+1 / 2} x^{1 / 2}+x^{n-k-1 / 2} x^{-1 / 2}
$$

and for $0 \leq k \leq[n / 2]-1, x^{k+1 / 2}$ and $x^{n-k-1 / 2}$ are in the same order as $x^{-1 / 2}$ and $x^{1 / 2}$. It follows that if we set 


$$
a_{i}=\left\{\begin{array}{l}
x^{i}+x^{n-i} \text { if } i<n / 2 \\
\frac{x^{i}+x^{n-i}}{2} \text { if } n \text { is even and } i=n / 2,
\end{array}\right.
$$

then $a_{0} \geq a_{1} \geq \ldots \geq a_{[n / 2]}$. If we also set

$$
b_{i}=\frac{1}{n+1}-\frac{1}{2^{n}}\left(\begin{array}{l}
n \\
i
\end{array}\right), \quad i=1,2, \ldots,[n / 2],
$$

it is clear that $b_{1} \geq b_{2} \geq \ldots \geq b_{[n / 2]}$; and since (1.1) - (1.3) are equalities when $a=b$, the sum of the $b_{i}$ is zero. (1.3) is then an immediate consequence of the following obvious lemma, which is also related to the rearrangements inequality.

LEMMA. If $k$ is a positive integer and $a_{1} \geq a_{2} \geq \ldots \geq a_{k} \geq 0$, and $b_{1} \geq b_{2} \geq \ldots \geq b_{k}$ and $b_{1}+b_{2}+\ldots+b_{k}=0$, then $a_{1} b_{1}+a_{2} b_{2}+\ldots+a_{k} b_{k} \geq 0$

COROLLARIES .

$$
\begin{aligned}
& x^{n}-1 \geq n(x-1)\left(\frac{x+1}{2}\right)^{n-1} ; x \geq 1 \text { and } n=0,1,2, \ldots \\
& x^{n}-1 \leq n(x-1)\left(\frac{x+1}{2}\right)^{n-1} ; 0 \leq x \leq 1 \text { and } n=0,1,2, \ldots
\end{aligned}
$$

Inequality (1.4) is a sharpening of Hardy, Littlewood, and Polya's (2.15.3) for part of the latter's range, while (1.5) is complementary to (2.15.3) for another part of its range. These are immediate consequences of (1.2), which is valid for all $x \geq 0$ by Theorem 1. (The case $n=0$ is not a consequence of Theorem 1. but is obvious; similarly (1.2) holds for $n=0$ if we interpret $1+x+x^{2}+\ldots+x^{n}$ as 1 for that case.)

$$
\begin{aligned}
& \text { Setting } x=1+t \text { in }(1.4) \text { and (1.5) gives us the inequalities } \\
& (1+t)^{n} \geq 1+n t\left(1+\frac{t}{2}\right)^{n-1} ; t \geq 0 \text { and } n=0,1,2, \ldots .
\end{aligned}
$$

and

$$
\begin{aligned}
& (1+t)^{n} \leq 1+n t\left(1+\frac{t}{2}\right)^{n-1} ;-1 \leq t \leq 0 \text { and } n=0,1,2 \ldots \\
& \text { Putting }-t \text { in place of } t \text { gives an alternative form: }
\end{aligned}
$$




$$
(1-t)^{n} \leq 1-n t\left(1-\frac{t}{2}\right)^{n-1} ; 0 \leq t \leq 1 \text { and } n=0,1,2, \ldots \text {. }
$$

Replacing $t$ by $1 / t$ in (1.6) gives us

$$
(t+1)^{n} \geq t^{n}+n\left(t+\frac{1}{2}\right)^{n-1} ; t \geq 0 \text { and } n=0,1,2, \ldots \text {. }
$$

This is better than (1.6) for $t>1$ though not as good for $t<1$ (for $n>2$ ). Similarly, from (1.8) we obtain :

$$
(t-1)^{n} \leq t^{n}-n\left(t-\frac{1}{2}\right)^{n-1} ; t \geq 1 \text { and } n=0,1,2, \ldots \text {. }
$$

(1.6) is a sharpening of the observation that compound interest beats simple interest: a period rate $t$, compounded for $n$ periods, is better than simple interest at the rate $t(1+t / 2)^{n-1}$.

(1.6) - (1.10) were obtained from (1.4) or (1.5) by invertible bilinear changes of variable; similarly each of (1.4) and (1.5) may be obtained from the other by replacing $\mathrm{x}$ by $1 / \mathrm{x}$. Thus $(1.4)-(1.10)$ are equivalent, and equivalent to Theorem 1 . Another bilinear transformation - setting $x=(1+t) /(1-t)$ in $(1.4)$ - gives us

$$
(1+t)^{n}-(1-t)^{n} \geq 2 n t ; 0 \leq t \leq 1 \text { and } n=0,1,2, \ldots \text {. }
$$

which is obvious! This provides a quick alternate proof of Theorem 1., but one which does not show the connection with the rearrangements inequality.

The form of inequalities (1.4) - (1.11) suggests consideration of non-integral $\mathrm{n}$. The inequalities are equivalent in this precise sense; for any given $n$, one of these inequalities holds for its stated range of values of the other variable $(x$ or $t$ ) if and only if all the other inequalities hold, for that $n$, for their stated ranges of the other variable. Thus for each $\mathrm{n}$ we may choose which of (1.4)

- (1.11) to study, at our convenience.

For $0<\mathrm{n}<1$ (and $0<t<1$ ) the binomial expansion of $(1+t)^{n}-(1-t)^{n}$ is

$$
2 n+2\left\{\left(\begin{array}{c}
n \\
3
\end{array}\right) t^{3}+\left(\begin{array}{c}
n \\
5
\end{array}\right) t^{5}+\ldots\right\},
$$


and each of the coefficients $\left(\frac{n}{3}\right),\left(\begin{array}{l}n \\ 5\end{array}\right), \ldots$ is positive. It follows that (1.11) holds for $\mathrm{n}$ between 0 and 1 .

For $\mathrm{n}$ between 1 and 2 , all of $\left(\begin{array}{l}\mathrm{n} \\ 3\end{array}\right),\left(\begin{array}{l}\mathrm{n} \\ 5\end{array}\right), \ldots$ are negative. So for $1<\mathrm{n}<2$ (and in fact $1 \leq \mathrm{n} \leq 2$ ), (1.11) holds with the inequality reversed. For non-integral $n>2$ we look at (1.6). Set

$$
f(t, n)=\frac{1+n t\left(1+\frac{t}{2}\right)^{n-1}}{(1+t)^{n}}
$$

$f(0, n) \equiv 1$. To show that $(1.6)$ holds, it is sufficient to show that

$$
\frac{\partial f}{\partial t}(t, n)=\frac{n}{(1+t)^{n+1}}\left\{\left(1+\frac{2-n}{2} t\right)\left(1+\frac{t}{2}\right)^{n-2}-1\right\}
$$

is $\leq 0$ for $t \geq 0, n \geq 2$. Setting $s=n-2$, the quantity in brackets is

$$
\left(1-\frac{s t}{2}\right)\left(1+\frac{t}{2}\right)^{s}-1
$$

This is certainly negative when $1-s t / 2$ is negative; when the latter is nonnegative, the whole quantity is

$$
\leq\left(1-\frac{s t}{2}\right) \quad\left(e^{\frac{s t}{2}}\right)-1 \leq 0 .
$$

Summing up we have :

THEOREM 2. Each of the inequalities (1.4) - (1.11) holds also for non-integral $\mathrm{n}$, when $0<\mathrm{n}<1$ and when $\mathrm{n}>2$ : for $1<\mathrm{n}<2$ each of $(1.4)-(1.11)$ holds with the direction of the inequality reversed.

\section{REFERENCES}

1. Hardy, G.H., J.E. Littlewood and G. Polya, Inequalities, Cambridge University Press (1959). 


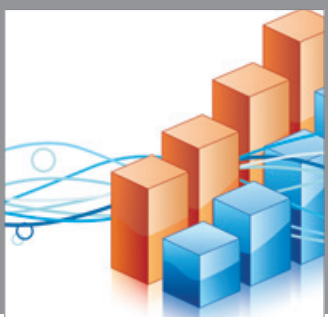

Advances in

Operations Research

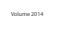

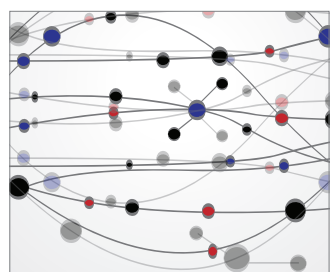

\section{The Scientific} World Journal
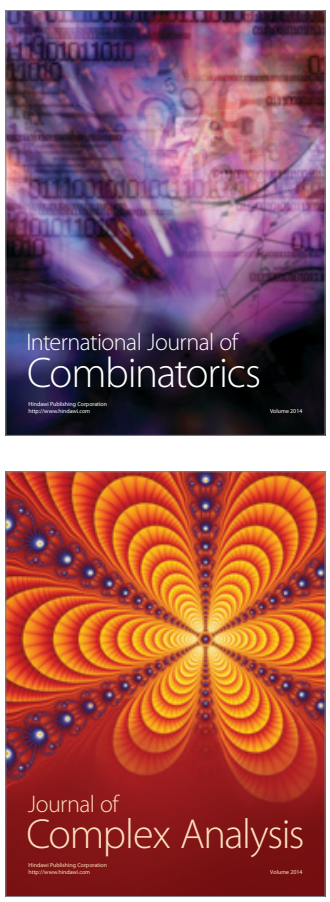

International Journal of

Mathematics and

Mathematical

Sciences
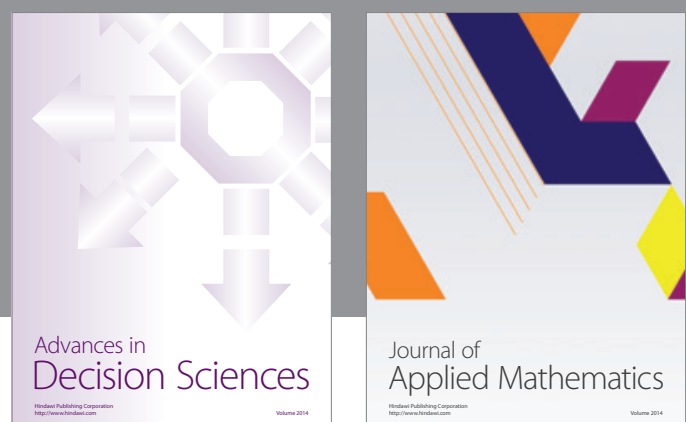

Journal of

Applied Mathematics
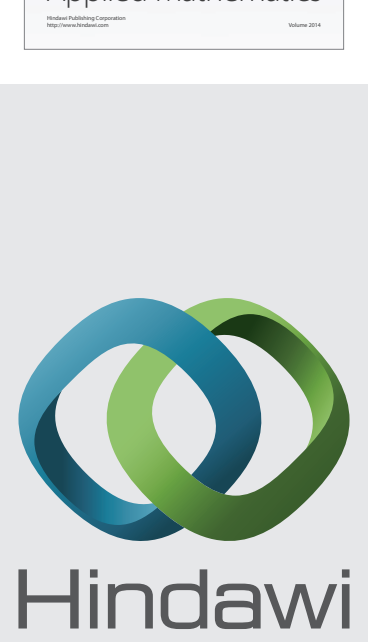

Submit your manuscripts at http://www.hindawi.com
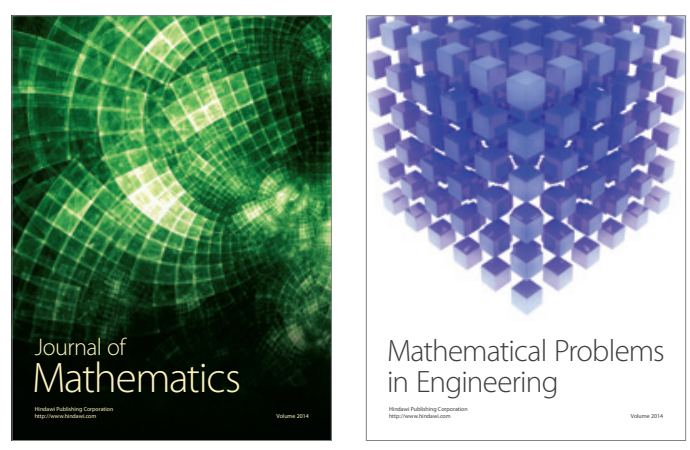

Mathematical Problems in Engineering
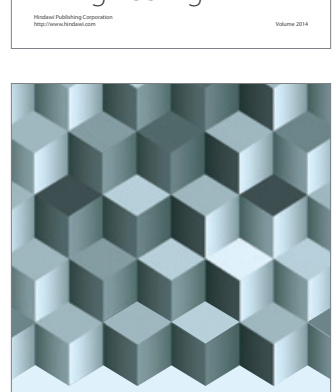

Journal of

Function Spaces
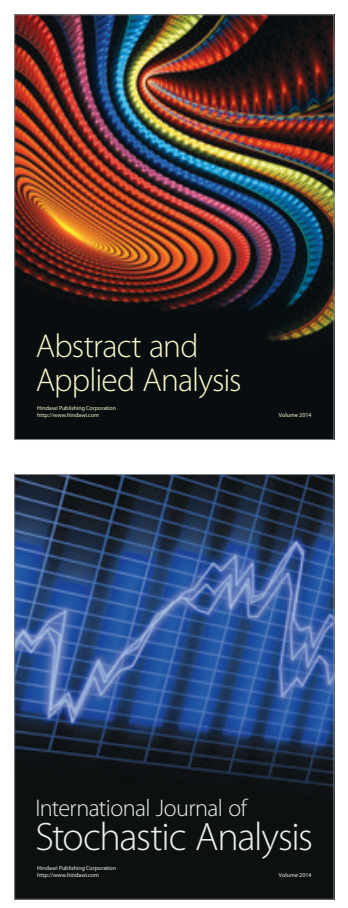

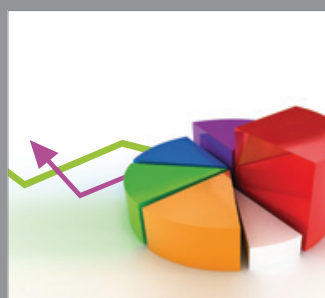

ournal of

Probability and Statistics

Promensencen
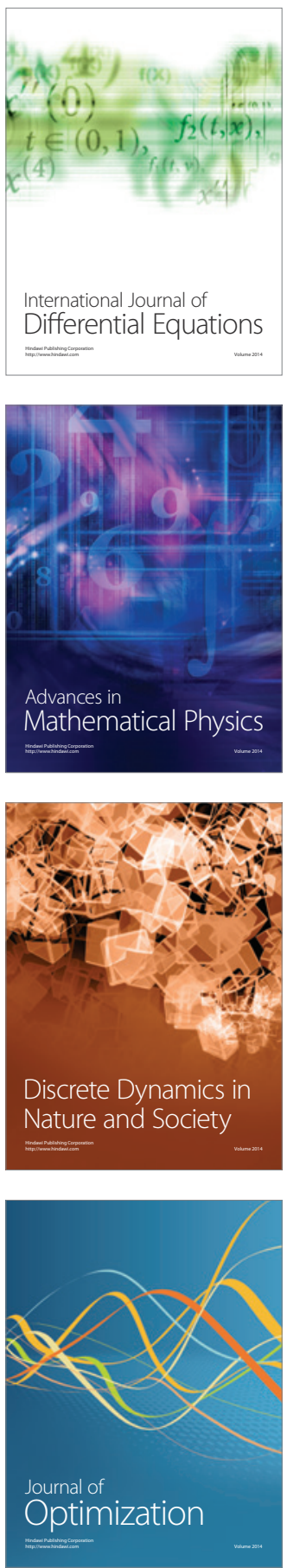